\title{
IMPAIRED RIGHT ATRIAL STRAIN IS ASSOCIATED WITH DECOMPENSATED HEMODYNAMICS IN PULMONARY ARTERIAL HYPERTENSION
}

\author{
Liang Zhong ${ }^{1,2}$, Shuang Leng ${ }^{1}$, Xiao-Dan Zhao ${ }^{1}$, Ju-Le Tan ${ }^{1,2}$, Ru-San Tan ${ }^{1,2}$ \\ ${ }^{1}$ National Heart Centre Singapore, 5 Hospital Drive, Singapore 169609 \\ ${ }^{2}$ Duke NUS Medical School, 8 College Road, Singapore 169857
}

The transition of right ventricle (RV) from a compensated to decompensated state contributes to survival in pulmonary arterial hypertension (PAH). However, little is known about the significance of right atrial (RA) dysfunction on disease progression in $\mathrm{PAH}$. In this context, there has been growing interest in markers of RA myocardial dysfunction. Speckle tracking echocardiography, which has been principally used to measure the myocardial strain, is technically challenging in the RA due to the thin atrial wall. Feature tracking cardiovascular magnetic resonance (FT-CMR) software designed to derive myocardial strain from CMR cine images has become available for measurements of atrial longitudinal strain. However, in subjects with relatively vigorous tricuspid annular motion, contour tracking of the RA free wall segment adjacent to the tricuspid valve is adversely affected and becomes the source of errors. In contrast to FT-CMR, we present a rapid assessable strain parameter that requires the automatic tracking of only 3 anatomical reference points thus avoiding the segment contour tracking near the insertion of the anterior leaflet into the tricuspid annulus.

Our rapid semi-automated (RSA) longitudinal strain is assessed by automatically tracking the distance $(L)$ between the right atrioventricular junction and a user-defined point at the mid posterior RA wall on standard CMR 4-chamber view (Fig. 1). The atrioventricular junctions (squares in Fig. 1) are selected as the tricuspid valve insertion points at the septal and free wall borders of the annulus on the 4-chamber view. ${ }^{1,2}$ The midpoint of posterior RA wall is defined as the intersection point of the RA posterior wall and the RA long-axis. The tracking system uses the method of adaptive template matching and is semi-automatic with mask selection in the initial frame as the only user input. The calculation of RA longitudinal strain $(\varepsilon)$ at any time point $(t)$ in the cardiac cycle from the time of minimal RA volume (time 0 ) is based on the Lagrange strain formula as follows: $\varepsilon(t)=\left(L(t)-L_{0}\right) \times 100 / L_{0}$. Three phasic strain measurements are derived (Fig. 1): total strain $\left(\varepsilon_{s}\right.$, corresponding to atrial reservoir function), passive strain ( $\varepsilon_{e}$, corresponding to atrial conduit function), and active strain ( $\varepsilon_{a}$, corresponding to atrial booster pump function). Accordingly, three strain rate (SR) measurements are evaluated by taking the first-order derivative of the strain data (Fig. 1): total strain rate $\left(S R_{s}\right)$, passive strain rate $\left(S_{e}\right)$, and active strain rate $\left(S R_{a}\right)$. Strain and $S R$ parameters from the RV septal and free wall of the 4-chamber view are averaged to obtain mean results for analysis.

The RSA method was applied in a group of $80 \mathrm{PAH}$ patients, including 58 with hemodynamically compensated RV function (PAH-C) and 22 with decompensated RV function (PAH-D), and 80 age- and gender-matched normal controls. In PAH compared to controls, significantly reduced RA strains and SRs were observed. Among PAH patients, PAH-D had significantly impaired RA strains and SRs compared to PAH-C. $R A$ total stain and passive strain were the best parameters for differentiating PAH-D from PAH-C. Reduced RA strains were significantly associated with higher risk of clinical worsening in PAH. RA passive strain was the best predictor of a composite adverse event endpoint (Harrell's C-statistic, 0.75; hazard ratio, $0.84 ; P=0.019$ ) compared to other conventional RA and RV functional measurements. RA strain parameters investigated, which are highly reproducible and readily obtainable from post-processing of standard 4-chamber cine CMR images, are promising candidate indices for non-invasive detection of RA dysfunction, RV decompensation and monitoring of disease progression in PAH patients.

\section{References:}

1. Leng S, Jiang M, Zhao XD, Allen JC, Kassab GS, Ouyang RZ, Tan JL, He B, Tan RS, Zhong L. Three-dimensional tricuspid annular motion analysis from cardiac magnetic resonance feature-tracking. Ann Biomed Eng. 2016;44:35223538.

2. Leng S, Tan RS, Zhao XD, Allen J, Koh AS, Zhong L. Validation of a rapid semi-automated method to assess left atrial longitudinal phasic strains on cine cardiovascular magnetic resonance imaging. J Cardiovasc Magn Reson. 2018;20:71. 


\section{Rapid semi-automated strain and strain rate}
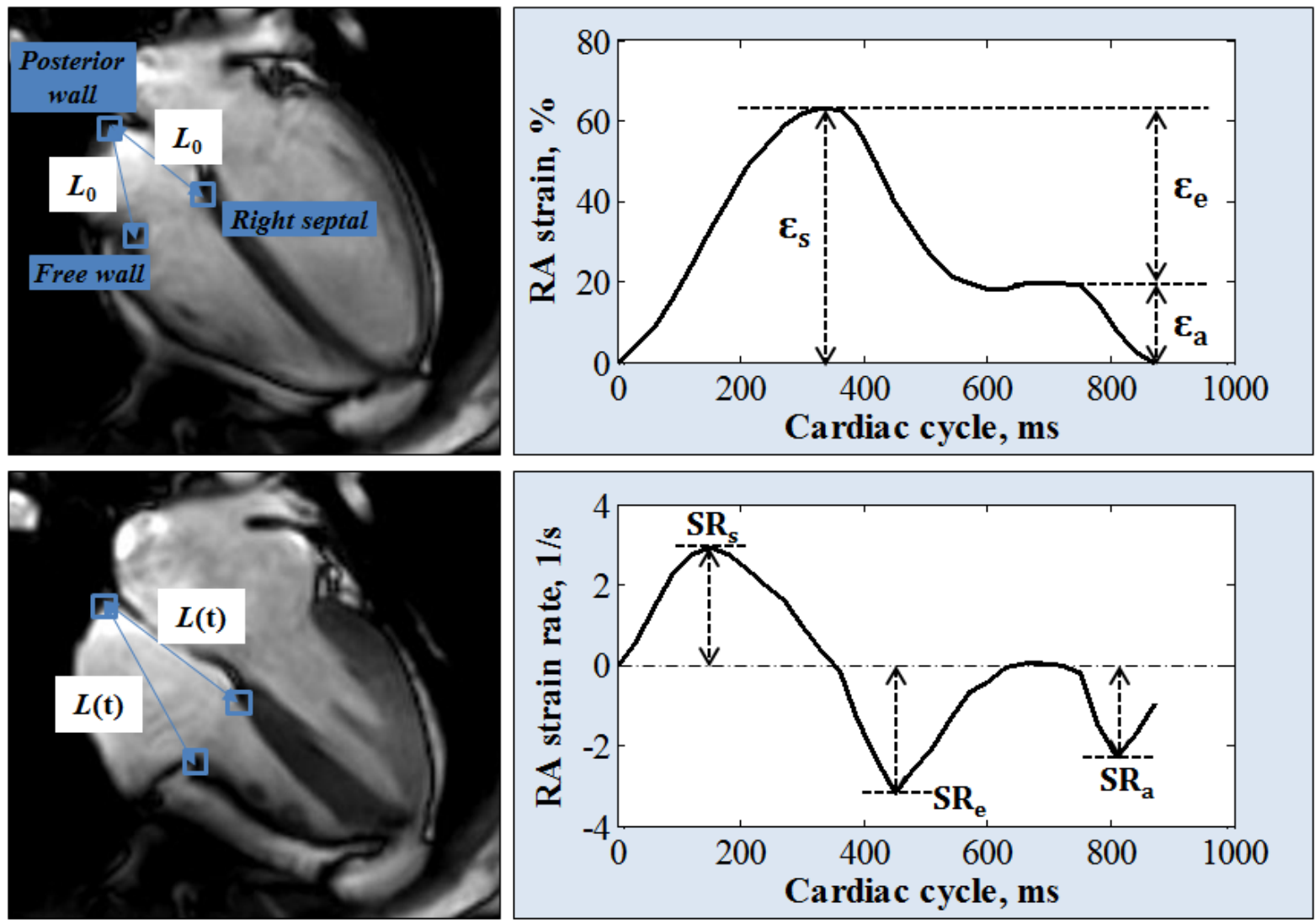

Figure 1. Rapid semi-automated right atrial strain and strain rate measurement in 4-chamber view. $L$ denotes the distance between the right atrioventricular junction (right septal and free wall) and a user-defined point at the mid posterior right atrial wall on standard cardiovascular magnetic resonance 4-chamber view. Rapid semiautomated strain was derived from time variation of distance $L$. Total strain $\left(\varepsilon_{s}\right)$ and strain rate $\left(\mathrm{SR}_{\mathrm{s}}\right)$ correspond to reservoir function. Passive strain $\left(\varepsilon_{e}\right)$ and strain rate $\left(\mathrm{SR}_{e}\right)$ correspond to conduit function. Active strain $\left(\varepsilon_{a}\right)$ and strain rate $\left(\mathrm{SR}_{\mathrm{a}}\right)$ correspond to booster pump function. 